augmented by central pain mechanisms, including central sensitisation. Measures of the level of central involvement in pain could inform clinical decision making. Self-report characteristics of depression, anxiety, cognitive difficulties catastrophizing, sleep disturbance, fatigue, and widespread pain distribution together contribute to a Central Mechanisms Trait which is associated with central sensitisation and OA knee pain

Objectives: Using self-report questionnaire data from the Osteoarthritis Initiative Cohort Study (OAI) we aimed to evaluate the prognostic performance of baseline CMT for pain at 24-months.

Methods: OAI participants with knee OA or at risk of knee OA with pain in the same knee at both index time point (48-months) and one year prior to that date were included $(n=1984)$. Knee pain was measured using the Western Ontario and McMasters Universities Osteoarthritis Index (WOMAC) pain sub-scale, by reference to the index knee (the knee with the highest WOMAC pain sub-scale score at baseline). Questionnaire items were selected to assess the 7 available characteristics identified by Akin-Akinyosoye et al.[1], from which a single CMT factor was calculated by confirmatory factor analysis. Anxiety, fatigue and cognitive difficulties were assessed by single items, depression and sleep disturbance represented by multiple items, and catastrophising by using the Coping Strategies Questionnaire - Catastrophising sub-scale. Pain distribution was defined as a sum of other painful joints at or below the hip. A CMT factor was derived from the 7 characteristics using confirmatory factor analysis. The association between the CMT factor score and 24-month pain (adjusted for baseline pain radiographic $\mathrm{OA}$ (Kellgren-Lawrence $(\mathrm{KL})$ scale) and demographic confounders) was investigated using generalised linear regression with a negative binomia link function.

Results: At baseline, participants had a mean (SD) age 65(9) years, a BMI $29.6(5.1) \mathrm{kg} / \mathrm{m}^{2}, 60 \%$ were female, $19.8 \%$ were African American, $\mathrm{KL}$ score was $1.92(1.35)$ indicating that the majority of the cohort had radiographic OA. Model diagnostics informed the CMT model, with the final model having an RMSEA of $0.073(90 \% \mathrm{Cl}$ 0.070-0.076). Data were consistent with a single factor model for CMT. In the multivariable model, higher baseline CMT scores were significantly associated with 24-month WOMAC pain scores, with or without adjustment for baseline pain and other covariates, including $\mathrm{KL}$ score (multivariable model; std beta $=0.173(\mathrm{SE}=0.027), \mathrm{p}=0.004)$. Association of baseline CMT was of similar strength, and over and above association of $\mathrm{KL}$ score with 24-month pain (std beta $=0.164$ ( $S E=0.038), p=<0.001$ ). Adjusted regression coefficients and associated $p$-values are shown in Table 1.

Table 1. Adjusted regression coefficients for analysed variables against WOMAC pain at 24-months

\begin{tabular}{lll}
\hline Variables & Std beta (SE) & $\mathrm{P}$ \\
\hline Sex & $-0.096(0.101)$ & 0.344 \\
Age, y & $-0.001(0.006)$ & 0.881 \\
$\mathrm{BMI}, \mathrm{kg} / \mathrm{m}^{2}$ & $0.017(0.010)$ & 0.088 \\
Index Knee Kellgren-Lawrence Score & $\mathbf{0 . 1 6 4 ( 0 . 0 3 8 )}$ & $<0.001$ \\
CMT Factor Score & $\mathbf{0 . 1 7 3 ( 0 . 0 6 0 )}$ & $\mathbf{0 . 0 0 4}$ \\
Baseline Pain & $\mathbf{0 . 8 5 7 ( 0 . 0 3 5 )}$ & $<0.001$
\end{tabular}

$n=1421$, rows in bold indicate significant association $(p<0.05)$, associations adjusted for race and ethnicity

Conclusion: CMT predicts worse pain prognosis with a similar magnitude to radiographic $\mathrm{OA}$ even after adjustment for other factors. A self-report tool which included items relevant to the characteristics included in the CMT may help to select people with OA knee pain with unfavourable pain prognosis. Poor outcomes related to central pain mechanisms or to joint structural damage might be amenable to treatments addressing central or peripheral pain mechanisms respectively.

REFERENCES:

[1] Akin-Akinyosoye et al., PAIN, 2018. 159(6): p. 1035-1044.

Acknowledgements: This abstract was prepared using an Osteoarthritis Initiative (OAI) public use data set and does not necessarily reflect the opinions or views of the OAl investigators, the $\mathrm{NIH}$, or the private funding partners. The authors wish to thank the participants, principal investigators, co-investigators and staff of all the hospitals who have contributed data to the OAI. The OAI is a public-private partnership comprised of five contracts (N01-AR-2-2258; N01-AR-2-2259; N01-AR-2-2260; N01-AR-2-2261; N01-AR-2-2262) funded by the National Institutes of Health, a branch of the Department of Health and Human Services, and conducted by the OAI Study Investigators. Private funding partners include Merck Research Laboratories; Novartis Pharmaceuticals Corporation,
GlaxoSmithKline; and Pfizer, Inc. Private sector funding for the OAI is managed by the Foundation for the National Institutes of Health.

Disclosure of Interests: Samuel Holden: None declared, Daniel McWilliams Grant/research support from: Pfizer and Eli Lilly, Stephanie Smith: None declared, David Walsh Consultant of: Pfizer, Eli Lilly, AbbVie and GlaxoSmithKline, Grant/research support from: Pfizer and Eli Lilly

DOI: 10.1136/annrheumdis-2021-eular.1595

\section{OP0085 ALTERED EXPRESSION OF NEUROTROPHINS AND THEIR RECEPTORS IN THE SKIN OF PATIENT WITH COMPLEX REGIONAL PAIN SYNDROME (CRPS)}

S. Stroeve ${ }^{1,2}$, S. Dudli ${ }^{1,2}$, I. Kolm ${ }^{3}$, I. Heggli ${ }^{1,2}$, N. Herger ${ }^{1,2}$, S. Catanzaro ${ }^{4}$, A. Schweizer ${ }^{5}$, M. Calcagni ${ }^{6}$, O. Distler ${ }^{1,2}$, F. Brunner $^{2}$, A. Juengel ${ }^{1,2}$ on behalf of Experimental Rheumatology Balgrist Campus. ${ }^{1}$ Center of Experimental Rheumatology, Department of Rheumatology, University Hospital Zurich, Zürich, Switzerland; ${ }^{2}$ Department of Physical Medicine and Rheumatology, Balgrist University Hospital, University of Zurich, Zurich, Switzerland; ${ }^{3}$ University Hospital Zurich, Department of Dermatology, Zurich, Switzerland; ${ }^{4}$ Balgrist University Hospital, University of Zurich, Switzerland, Unit of Clinical and Applied Research, Zurich, Switzerland; ${ }^{5}$ Balgrist University Hospital, University of Zurich, Switzerland, Department of Hand surgery, Zurich, Switzerland; ${ }^{6}$ University Hospital Zurich, Department of Plastic Surgery and Hand Surgery, Zurich, Switzerland

Background: Complex regional pain syndrome (CRPS) is a rare painful condition that usually appears after trauma or surgery of the extremities. Symptoms include pain, sensory, sudomotor and vasomotor disturbances, trophic changes and impaired motor function. The course varies from mild to chronic disease with a high impact on daily functioning and quality of life. In skin tissue, sustained inflammatory, fibrotic processes together with reduced epidermal nerve fibers are reported. Neurotrophins and their receptors are mediators in cell-to-cell communication and key mediators of pain signaling

Objectives: The aim of this study was to identify differential expression of neurotrophins and their receptors in the skin and skin fibroblasts of patients with CRPS Methods: Healthy controls $(\mathrm{HC})$ and patients with acute CRPS with symptoms for less than 6 months fulfilling the Budapest criteria were recruited. Pain scores were evaluated by numeric rating scale $(0=$ no pain, $100=$ maximal) and body perception was assessed using the Bath Body Perception Disturbance Scale (BBPDS) $(0=$ no perception disturbance, $57=$ maximal perception disturbance)

Skin biopsies of the affected and/or non-affected side were taken. Immunohistochemistry on formalin-fixed, paraffin-embedded skin tissue slides was used to show NT3 expression in skin tissues. Blinded analysis was done by an experienced dermato-pathologist determined staining graduated by $0=$ none $1=$ sparse, $2=$ moderate, $3=$ dense.

Skin fibroblast were isolated from skin biopsies by outgrowth cultures (CRPS, affected side, $n=6$ and $H C, n=5$ ). Cells (passage $3-6$ ) were starved and subsequently stimulated with TNF $\alpha(10 \mathrm{ng} / \mathrm{ml})$ or TGF $\beta(10 \mathrm{ng} / \mathrm{ml})$ for $24 \mathrm{~h}$ to mimic active disease and total RNA was isolated by miRNeasy kit. Gene expression of neurotrophins (NGF, BDNF, and NT3) and neurotrophin receptors (NGFR, TrkA TrkB and TrkC) was measured by quantitative real time PCR and quantified using the $\triangle \Delta C q$ method with GAPDH as a reference gene. ELISA was used to analyze NT3 protein expression in cell culture supernatants.

Results: In 5 of 9 patients with CRPS immunohistological staining of NT3 showed an higher expression (from low to moderate) in the affected side versus the non-affected side. In 4 of 9 patients the expression of NT3 was high in the non-affected side (moderate/dense) and stayed high in the affected side.

Of interest, the patients with increasing expression of NT3 in the affected side showed increased pain scores (max pain $80+/-10.95, n=5$ versus $48.16+/-18.16$ $n=4, p=0.059$ and changed body perception $26.8+/-8.68 n=5$ versus $6.5+/-3.91$, $\mathrm{n}=4, \mathrm{p}=0.016$ ).

Isolated skin fibroblasts from the affected side of patients with CRPS compared to healthy skin fibroblasts showed higher basal gene expression of NT3 $\left(\log _{2}\right.$ fold-change $\left.=1.9+/-0.4, p=0.005\right)$ and NGFR $\left(\log _{2}\right.$ fold-change $=3.6+/-$ $2.1, p=0.014)$. TNF $\alpha$ stimulated CRPS skin fibroblasts showed higher expression for NT3 $\left(\log _{2}\right.$ fold-change $\left.=2.1+/-1.2, p=0.002\right)$ compared to HC. TGFb stimulated skin fibroblasts of patients with CRPS showed higher expression of NT3 $\left(\log _{2}\right.$ fold-change $\left.=1.4+/-0.8, p=0.019\right), N G F R\left(\log _{2}\right.$ fold-change $=2.6$ $+/-1.8, p=0.036)$ and TrkC $\left(\log _{2}\right.$ fold-change $\left.=2.3+/-1.8, p=0.032\right)$ compared to $\mathrm{HC}$.

On protein level, NT3 showed a tendency of upregulation in unstimulated fibroblasts from CRPS patients comparing to HC (CRPS mean $=8.0+/-2.2 \mathrm{pg} / \mathrm{ml}, \mathrm{HC}$ 
mean $=6.3+/-1.8 \mathrm{pg} / \mathrm{ml}, p=0.25)$. After TNF $\alpha$ stimulation, protein level of NT3 was significantly higher in CRPS skin fibroblasts (CRPS mean $=10.6+/-2.4 \mathrm{pg} /$ $\mathrm{ml}, \mathrm{HC}$ mean $=4.8+/-1.3 \mathrm{pg} / \mathrm{ml}, p=0.004$ ).

Conclusion: These data indicate a new role of skin fibroblasts in CRPS. Differential basal and stimulated expression of NT3, the receptor for NT3 (TrkC) and NGFR, the common receptor for all neurotrophins, indicates deregulated communication of fibroblasts with the sensory nerve fibers in CRPS. This might contribute to the dysregulated healing process and sustained pain.

Disclosure of Interests: Sanne Stroeve: None declared, Stefan Dudli: None declared, Isabel Kolm: None declared, Irina Heggli: None declared, Nick Herger: None declared, Sabrina Catanzaro: None declared, Andreas Schweizer: None declared, Maurizio Calcagni Speakers bureau: Arthrex, Consultant of: Medartis, Arthrex, SilkBiomaterials, Grant/research support from: Medartis, Oliver Distler: None declared, Florian Brunner: None declared, Astrid Juengel: None declared DOI: 10.1136/annrheumdis-2021-eular.830

\section{OP0086 GENDER INFLUENCE ON CLINICAL MANIFESTATIONS, DEPRESSIVE SYMPTOMS AND BRAIN-DERIVED NEUROTROPHIC FACTOR (BDNF) SERUM LEVELS IN PATIENTS AFFECTED BY FIBROMYALGIA}

C. Gioia ${ }^{1}$, B. Lucchino ${ }^{1}$, C. Iannuccelli', G. Dolcini ${ }^{1}$, M. DI Franco ${ }^{1}{ }^{1}$ Sapienza Università di Roma, Reumatologia, Dipartimento di Scienze Cliniche, Internistiche, Anestesiologiche e Cardiovascolari, Sapienza Università di Roma, Roma, Italy

Background: Fibromyalgia (FM) is a common rheumatic disease characterized by chronic widespread pain, sleep and mood disorders. A higher prevalence of FM in women compared with men is well known, although the specific differences in clinical manifestations related to gender are still poorly defined. Brain-Derived Neurotrophic Factor (BDNF) is an endogenous growth factor that gained attention for its potential as biomarker of several diseases, including FM and depression.

Objectives: The aims of this study were to investigate gender-related difference among males and females affected by FM in clinical manifestations, depressive features and BDNF serum level, evaluating also the diagnostic potential of the latter.

Methods: We consecutively enrolled adult patients affected by FM (ACR 2016) referring to our out-patient clinic. Each subject underwent clinical and answered to questionnaires for the severity of FM symptoms (Revised Fibromyalgia Impact Questionnaire, R-FIQ) and depressive symptoms (Beck Depression Inventory-II, BDI-II). We collected blood samples from a subgroup of patients of both sexes, matched for age, for BDNF serum level dosage through ELISA. BDNF levels were assessed also in a control group, matched for sex and age.

Results: The cohort was composed by $201 \mathrm{FM}$ patients (172 F, 29M), mean age 49.13. Females showed higher values of R-FIQ total score $(p=0,0005)$ as well the specific items of the R-FIQ for pain $(p=0,013)$, fatigue $(p=0,014)$, memory problems $(p=0,007)$, tenderness to touch $(p<0,0001)$, balance problems $(p<0,0001)$ and sensitivity to environmental stimuli $(p=0,012)$ when compared with males (fig. 1). There was no difference in BDI-II between males and females, but notably male patients reported a significantly higher frequency of coexisting depressive disorder ( $p=0,038)$ (fig. 2). Serum BDNF levels were evaluated in $40 \mathrm{FM}$ patients and 40 healthy controls (HC) (F:M 1:1). BDNF levels were significantly lower in FM patients compared with $H C(p<0,0001)$. Among FM patients, BDNF levels were lower in males compared with females $(p<0,0001)$ (fig.3). BDNF did not correlate with any clinical and clinimetric parameter. BDNF showed a good diagnostic performance ( $A \cup C=0,89$, $\mathrm{Cl} 95 \%=0,82-0,9630, p<0,0001$ ) (fig. 4). At a cut-off value $<6,47 \mathrm{ng} / \mathrm{dl}$, BDNF showed a specificity of $75 \%$ and a sensibility of $92,31 \%,(\mathrm{Cl} 95 \%=79,68-97.35)$ for FM identification ( $\mathrm{LR}=3,692)$.

Conclusion: FM clinical manifestations are strongly dependant from gender. While females present a more severe disease and a higher burden of symptoms, mood disorders tend to be a major characteristic of males with FM. Reduced BDNF serum levels have been reported as typical of depressive disorders. Our findings of lower BDNF levels in male FM patients compared to females support this hypothesis. BDNF have potential as biomarker of the disease and should be validated in larger cohorts.

REFERENCES:

[1] Sarzi-Puttini et al. Nature Reviews 2020

[2] Colucci-D'Amato et al. Int J Molecular Sciences 2020

[3] Nugraha et al. Rheumatol Int 2012
[4] Schmitt et al. Ann Med 2016

[5] Melchior et al. Neuroscience 2016

[6] Stefani et al. Neuroscience Letters 2012
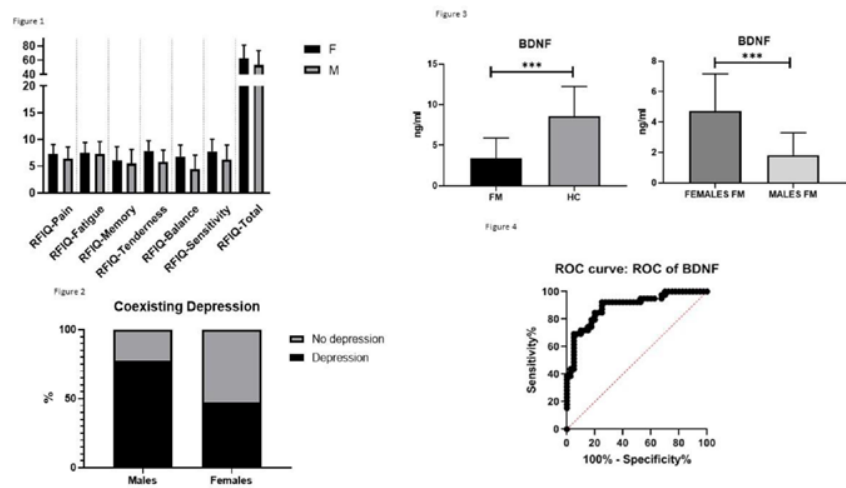

Disclosure of Interests: None declared DOI: 10.1136/annrheumdis-2021-eular.3326

\section{\begin{tabular}{|l|l}
\hline OP0087 COMORBIDITIES IN HAND OSTEOARTHRITIS \\
\hline
\end{tabular} PATIENTS: PREVALENCE AND IMPACT ON PAIN AND PAIN SENSITIZATION}

E. Mulrooney ${ }^{1}$, T. Neogi ${ }^{2}$, H. Solveig Dagfinrud ${ }^{1,3}$, H. Berner Hammer ${ }^{1,4}$, P. Steen Pettersen ${ }^{1}$, T. L. Gaarden ${ }^{5,6}$, K. Engedal ${ }^{7,8}$, T. K. Kvien ${ }^{1,9}$, K. Magnusson ${ }^{10}$, I. K. Haugen ${ }^{1} .^{1}$ Diakonhjemmet Hospital, Division of Rheumatology and Research, Oslo, Norway; ${ }^{2}$ Boston University School of Medicine, Section of Rheumatology, Boston, United States of America; ${ }^{3}$ University of Oslo, Department for Interdisciplinary Health Sciences, Oslo, Norway; ${ }^{4}$ University of Oslo, Department of Orthopaedics, Oslo, Norway; ${ }^{5}$ Diakonhjemmet sykehus, Alderspsykiatrisk Avdeling, Os/o, Norway; ${ }^{6}$ University of Oslo, Faculty of Medicine, Oslo, Norway;

${ }^{7}$ Norwegian Centre of Ageing and Health, Norwegian Centre of Ageing and Health, Oslo, Norway; ${ }^{8}$ University of Oslo, Department of Geriatric Medicine, Oslo, Norway; ${ }^{9}$ University of Oslo, Division of Orthopaedic Surgery, Oslo, Norway; ${ }^{10}$ Lund University, Department of Clinical Sciences, Lund, Sweden

Background: Pain is a hallmark symptom of hand osteoarthritis (OA). A subset of people with hand OA display centrally driven pain characteristics. Pain is driven by person-related factors, but how comorbidities are related to the hand OA pain experience is undetermined. Changes in pain pathophysiology and pain levels by for instants low-grade inflammation, might explain the link.

Objectives: The purpose of this study was to determine whether the burden of comorbidities or the individual comorbidities were associated with pain and pain sensitization in persons with hand OA.

Methods: These cross-sectional analyses included 282 participants with hand OA from the Nor-Hand study. Comorbidities were assessed by an index (subscales: Disease, Limitation and Treatment, (0-45 scale)) by Sangha et al. The participants completed pain questionnaires; Numeric Rating Scale (NRS) hand pain (0-10) and all joints (0-10), Australian/Canadian (AUSCAN) hand pain subscale (0-20 scale), Western Ontario \& McMaster Universities Arthritis Index (WOMAC) pain subscale (0-20) and homunculus (painful joint sites last six weeks, (0-18)). Pressure pain threshold (PPT, handheld algometer, $\mathrm{kg} / \mathrm{cm}^{2}$ ) at tibialis anterior and trapezius and Temporal summation (TS) at the distal radioulnar joint tapped with weighted probe (x10), assessed central pain sensitization. TS-delta was calculated of the first and peak measure of the fifth or tenth tap. Linear regression was used to examine all relations, while adjusted for age, sex, body mass index and education.

Results: The participants (89\% women, median (IQR) age: 61 (57-66)) showed broad range in pain severity. Most frequent comorbidities were back pain $(n=170,60 \%)$, hypertension $(n=85,30 \%)$, stomach ulcer/abdominal disease $(n=62,22 \%)$ and depression $(n=46,16 \%) .281(99.3 \%)$ participants reported $>1$ 